OPEN ACCESS

Edited by: Martin G. Klotz,

Queens College, City University of New York, USA

Reviewed by:

Colin Murrell,

University of East Anglia, UK

Tobias Jürgen Erb,

ETH Zurich, Switzerland

*Correspondence:

Marina G. Kalyuzhnaya

mkalyuzhnaya@mail.sdsu.edu

Specialty section:

This article was submitted to Microbial Physiology and Metabolism,

a section of the journa

Frontiers in Microbiology

Received: 14 October 2016 Accepted: 23 December 2016

Published: 10 January 2017

Citation:

Demidenko A, Akberdin IR

Allemann M, Allen EE and Kalyuzhnaya MG (2017) Fatty Acid

Biosynthesis Pathways in

Methylomicrobium buryatense

5G(B1). Front. Microbiol. 7:2167.

doi: 10.3389/fmicb.2016.02167

\section{Fatty Acid Biosynthesis Pathways in Methylomicrobium buryatense 5G(B1)}

\author{
Aleksandr Demidenko ${ }^{1,2}$, Ilya R. Akberdin ${ }^{1}$, Marco Allemann ${ }^{2}$, Eric E. Allen ${ }^{2}$ and \\ Marina G. Kalyuzhnaya ${ }^{1 *}$ \\ 'Department of Biology, San Diego State University, Campanile Drive, San Diego, CA, USA, ${ }^{2}$ Scripps Institution of \\ Oceanography, University of California San Diego, Gilman Drive, La Jolla, CA, USA
}

Methane utilization by methanotrophic bacteria is an attractive application for biotechnological conversion of natural or biogas into high-added-value products. Haloalcaliphilic methanotrophic bacteria belonging to the genus Methylomicrobium are among the most promising strains for methane-based biotechnology, providing easy and inexpensive cultivation, rapid growth, and the availability of established genetic tools. A number of methane bioconversions using these microbial cultures have been discussed, including the derivation of biodiesel, alkanes, and OMEGA-3 supplements. These compounds are derived from bacterial fatty acid pools. Here, we investigate fatty acid biosynthesis in Methylomicrobium buryatense $5 G(B 1)$. Most of the genes homologous to typical Type II fatty acid biosynthesis pathways could be annotated by bioinformatics analyses, with the exception of fatty acid transport and regulatory elements. Different approaches for improving fatty acid accumulation were investigated. These studies indicated that both fatty acid degradation and acetyl- and malonyl-CoA levels are bottlenecks for higher level fatty acid production. The best strain generated in this study synthesizes $111 \pm 2 \mathrm{mg} / \mathrm{gDCW}$ of extractable fatty acids, which is $\sim 20 \%$ more than the original strain. A candidate gene for fatty acid biosynthesis regulation, farE, was identified and studied. Its deletion resulted in drastic changes to the fatty acid profile, leading to an increased pool of C18-fatty acid methyl ester. The FarE-regulon was further investigated by RNA-seq analysis of gene expression in farE-knockout mutants and farE-overexpressing strains. These gene profiles highlighted a novel set of enzymes and regulators involved in fatty acid biosynthesis. The gene expression and fatty acid profiles of the different farE-strains support the hypothesis that metabolic fluxes upstream of fatty acid biosynthesis restrict fatty acid production in the methanotroph.

Keywords: methane valorization, methanotrophs, fatty acid metabolism, farE, fatty acid elongation

\section{INTRODUCTION}

Methane bioconversion by methanotrophic bacteria is attracting attention from biotechnologists because of its inexpensiveness and the abundance of natural gas as its potential feedstock. However, economically effective examples of such bioconversions have been limited to just the production of single-cell protein and polyhydroxybutyrate products (Levett et al., 2016; Strong et al., 2016). 
Advancement in this area is limited by the paucity of approaches to manipulate the methanotroph genome and the even more limited knowledge of the genomic targets to be manipulated. We and others are developing genomics and genetic tools for methanotroph genome manipulation (Ojala et al., 2011; Puri et al., 2015; Henard et al., 2016). In this work we focus on identifying novel genomic targets-specifically, we studied fatty acid (FA) biosynthesis/degradation pathways and regulation as a means to increase cellular lipid content. Total FAs extracted from bacteria have been shown to be valuable precursors for production of liquid transportation fuels (Lennen and Pfleger, 2012). Furthermore, the interest in methanotrophic microbes as methane-driven microbial factories for the production of a variety of FA-based products, ranging from industrial surfactants and detergents to cosmetic carotenoid-based products and OMEGA supplements, is on the rise. Methanotrophic bacteria produce high levels of FAs for the synthesis of intracytoplasmic membranes (ICM, Patt and Hanson, 1978; Jahnke and Nicholst, 1986; Sessions et al., 2002). However, the metabolic pathways for FA biosynthesis have never been thoroughly investigated.

Methylomicrobium buryatense 5G(B1), a gammaproteobacterial methanotrophic bacterium, can use methane as a sole carbon and energy source. A set of unique properties of the strain, including its fast growth rate, its ability to grow over a broad range of salinity and $\mathrm{pH}$, and the availability of a draft genome, a validated flux balance model, and a set of genetic tools, make it an ideal model for studying lipid biosynthesis (Kaluzhnaya et al., 2001; Khmelenina and Beck, 2013; De la Torre et al., 2015; Puri et al., 2015; Henard et al., 2016). The metabolic pathways for methane utilization in $M$. buryatense $5 G(B 1)$ have been recently refined (De la Torre et al., 2015; Gilman et al., 2015). Methane utilization starts with the oxidation of methane to methanol by a particulate or soluble methane monooxygenase enzymes (Kaluzhnaya et al., 2001); methanol is then converted to formaldehyde by PQQ-dependent methanol dehydrogenases, a two subunit Ca-containing enzyme or a one subunit La-induced enzyme (Chu and Lidstrom, 2016; Chu et al., 2016). Formaldehyde is either oxidized to $\mathrm{CO}_{2}$ to supply energy or assimilated into sugars through a ribulose monophosphate (RuMP) pathway, a fraction of which is then converted through pyruvate to acetyl-CoA (Figure 1A), a building block for FA synthesis (Figure 1B). Similar to other methanotrophic bacteria, $M$. buryatense $5 G(B 1)$ produces significant amounts of ICMs, which can occupy up to $60 \%$ of its cellular volume (Collins and Kalyuzhnaya, unpublished data). Methanotroph membranes are composed of phosphatidylethanolamine- (63-95\%), phosphatidylglycerol- (2.5-18\%), and phosphotidylserine(2.2-7.6\%) linked $\mathrm{C}_{16}$ or $\mathrm{C}_{16: 1}$ FAs (Kaluzhnaya et al., 2001; Gilman et al., 2015). Here, we present a genomebased reconstruction of the FA biosynthesis pathways in $M$. buryatense $5 G(B 1)$. Assumptions from the analysis of genomic data were further supported by gene expression data and mutagenesis. A detailed search within the M. buryatense 5G(B1) genome revealed several candidate gene targets for strain engineering efforts to improve production of bulk FA-based chemicals.

\section{MATERIALS AND METHODS}

\section{Strains and Genetic Manipulations}

Strains and genetic constructs used in this study are listed in Table 1. Strain AP18 was chosen as the base strain for most of the genetic modifications described here. The strain descends from the $M$. buryatense $5 G(B 1)$ strain, a lab-adapted variant of wild type $M$. buryatense $5 \mathrm{G}$. The strain is resistant to rifampicin, has improved transformation efficiency, and lacks the $76 \mathrm{kB}$ plasmid, the glycogen synthase genes $g \lg A 1$ and $g \lg A 2$, and the sucrose-phosphate synthase (sps) gene (Puri et al., 2015). These modifications resulted in a very modest increase in the FA pool as well as an inability to produce glycogen and/or sucrose. So far no significant impacts on cell growth and methane consumption have been observed. The strain AP18 and plasmid constructs used to create acetate kinase-deleted and acetyl-CoA carboxylaseoverexpressing mutants were kindly provided by the Lidstrom Laboratory (University of Washington).

$M$. buryatense strains were grown on methane as described (Ojala et al., 2011), with some modification of the growth medium (Table S1). Escherichia coli strains were grown on Luria-Bertani media supplemented with kanamycin $(100 \mu \mathrm{g} / \mathrm{ml})$ and ampicillin $(100 \mu \mathrm{g} / \mathrm{ml})$. Genetic manipulations with $M$. buryatense were done as described (Puri et al., 2015). For unmarked gene deletions, the pCM433kanT plasmid carrying $\sim 600$-bp of sequences flanking the to-be-deleted genes was introduced. After conjugation, single-crossover kanamycinresistant $M$. buryatense clones were plated on rifampicin to counter-select against E. coli. Then, to select for Kan-sensitive double crossover clones with deleted genes of interest, singlecrossover clones were passaged on plates with $2.5 \%$ sucrose and the resulting colonies were PCR-genotyped for the absence of the gene of interest followed by sequencing. For overexpression of genes, the pAWP78 plasmid was used as a vector. All genes were cloned under the tac-promoter, which provides strong constitutive expression in M. buryatense (Puri et al., 2015). The nucleotide positions of the sequences cloned into plasmids are outlined in Table S2.

\section{Induction of Acetyl-CoA Carboxylase}

The acetyl-CoA carboxylase expression system (pAWP78::accABCD) was obtained from Lidstrom and Puri. The construct includes the native acetyl-CoA carboxylase genes (subunits A, B, C, and D) cloned into pAWP78 under the tetracycline-inducible pTet promoter (Puri, unpublished data). The plasmid was transformed into $E$. coli S17-1 and transferred into the 5GB1C, AP18, AP18 $\Delta$ ack, AP18 $\Delta$ fadABE $\Delta$ ack, abd $\mathrm{AP} 18 \triangle$ fadABE backgrounds via conjugation. Cells were grown until mid-exponential phase (at $\mathrm{OD} \sim 0.5$ ). Each cell line was represented by biological (3) and technical (2 per biological replicate) replicates. Expression of acetyl-CoA carboxylase was induced by addition of anhydrotetracycline $(1 \mu \mathrm{M}$ final) followed by a 6 -h incubation before collection of cells by centrifugation.

\section{FA Methyl Ester (FAME) Analyses}

Fifty milliliters of cell cultures grown at $30^{\circ} \mathrm{C}$ with $50 \mathrm{ml}$ of methane to $\mathrm{OD}_{600} \sim 1$ were collected by centrifugation, washed 


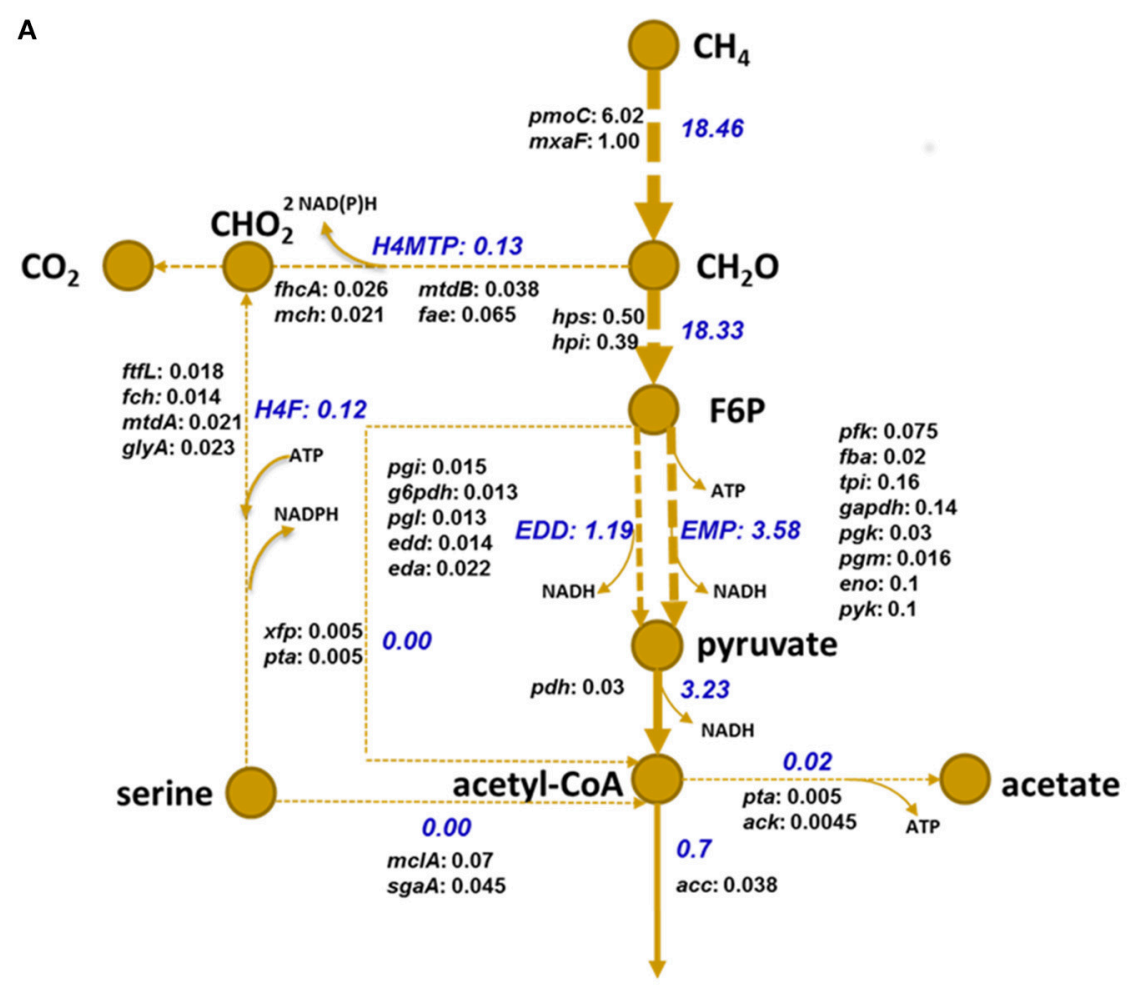

\section{FA biosynthesis}

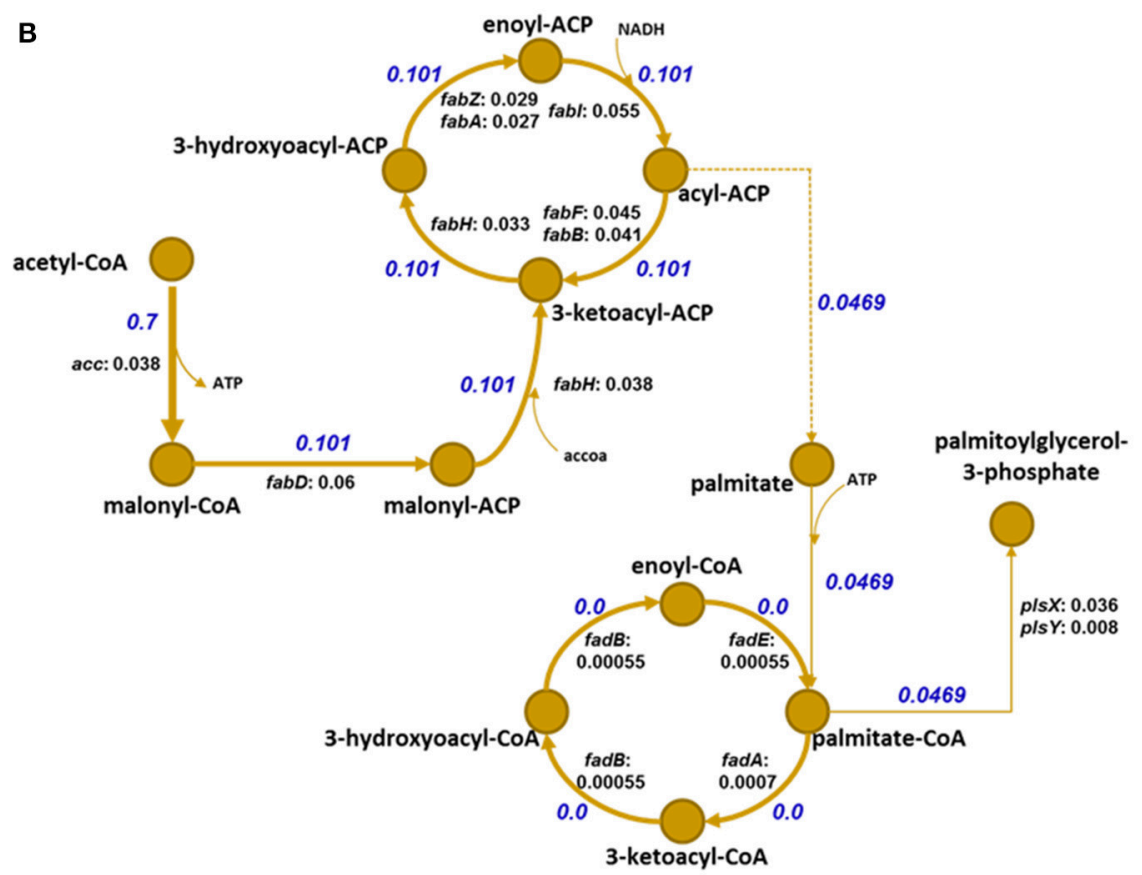

FIGURE 1 | Metabolic pathways for methane oxidation (A) and FA synthesis (B) in M. buryatense $5 G(B 1)$ based on a gene inventory study, transcriptomic data analysis, and genome-scale modeling (De la Torre et al., 2015). Numbers in blue represent that particular reaction's fluxes according to the developed genome-scale model (De la Torre et al., 2015); numbers in black after gene names denote abundances of respective transcript normalized to an abundance of methanol

dehydrogenase. Gene names coding enzymes of the methane assimilation pathways are extracted from BioCyc Database Collection (http://biocyc.org/). Enzyme EC numbers and corresponding gene IDs are listed in Table S5. Methane utilization starts from oxidation to methanol by methane monooxygenase enzyme in periplasm; 


\section{FIGURE 1 | Continued}

methanol is then metabolized to formaldehyde by a periplasmic pyrroloquinoline quinone-linked methanol dehydrogenase. Formaldehyde is either oxidized to $\mathrm{CO}_{2}$ or assimilated in cytoplasm through ribulose monophosphate (RuMP) and EMP/EDD (Embden-Meyerhof-Parnas/Entner-Doudoroff) pathways (A), fraction of which are then converted through pyruvate to acetyl-CoA, a precursor for FA synthesis (B). Designations: $\mathrm{CH}_{4}$, methane; $\mathrm{CH}_{2} \mathrm{O}$, formaldehyde; $\mathrm{CHO}_{2}$, formate; $\mathrm{H} 4 \mathrm{MTP}$, tetrahydromethanopterin pathway; $\mathrm{H} 4 \mathrm{~F}$, methylene tetrahydrofolate pathway; $\mathrm{CO}_{2}$, carbon dioxide; f6p, fructose 6-phosphate; acCoA, acetyl-CoA; $\mathrm{NADH}$, nicotinamide adenine dinucleotide reduced; NADPH, dihydronicotinamide adenine dinucleotide phosphate reduced; ATP, adenosine-triphosphate.

TABLE 1 | List of strains and plasmids used in this study.

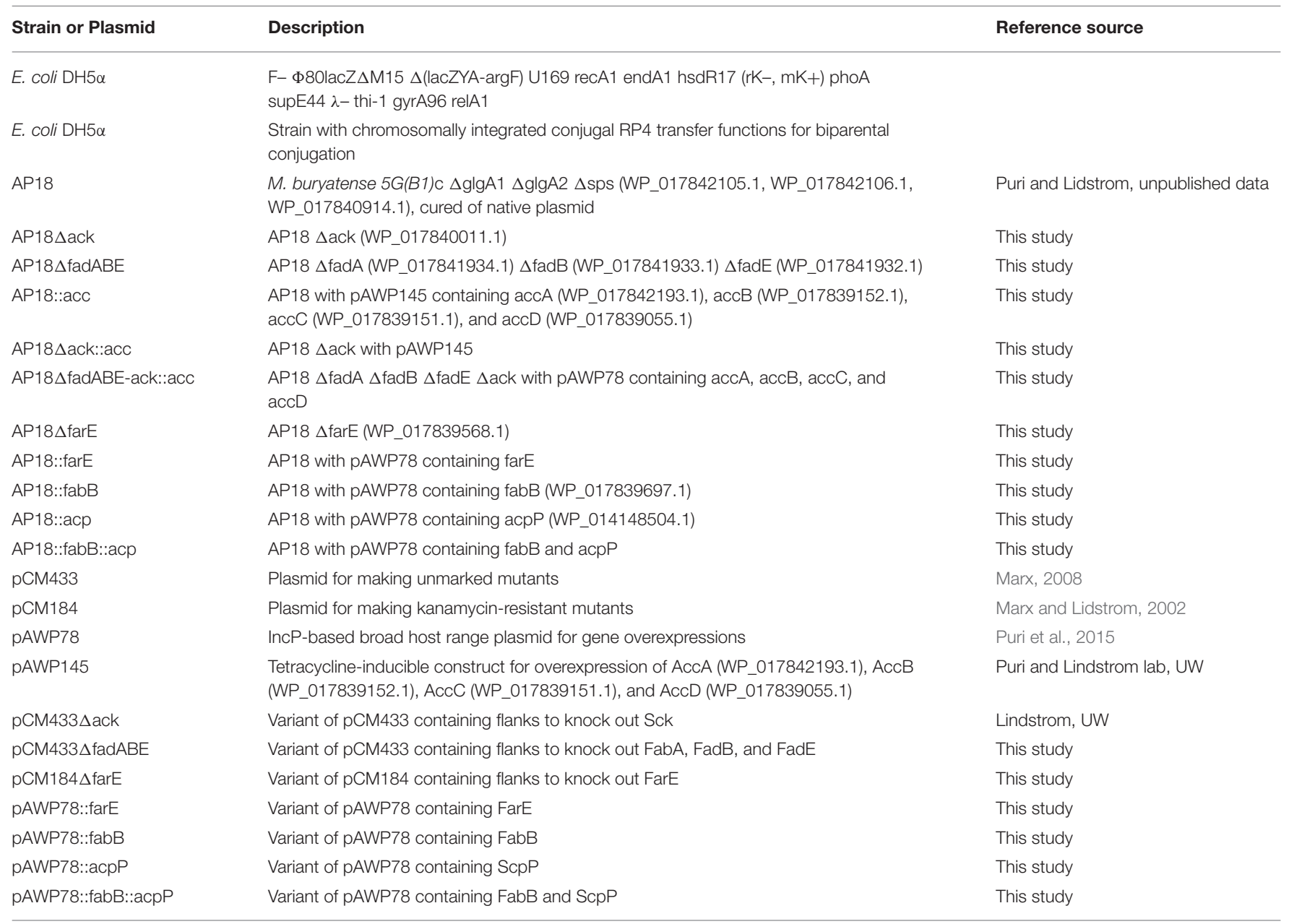

twice with $10 \mathrm{mM}$ Tris- $\mathrm{Cl}$ (pH8.0) and $50 \mathrm{mM}$ of $\mathrm{NaCl}$, and lyophilized. Biological replicates $(n=2 \div 10)$ were submitted to Matrix Genetics (Seattle, WA, http://matrixgenetics.com) for FAME derivatization and GC-MS analysis.

\section{RNA Sequencing}

Strains (i) AP18 (ii) AP18 $\Delta$ fabR, and (iii) AP18::pAWP78 (fabR) were grown in duplicate in a $50 \mathrm{ml}$ volume at $30^{\circ} \mathrm{C}$ with shaking with methane. RNA from pelleted cells was extracted according to (Griffiths et al., 2000) and treated with PureLink DNaseI (ThermoFisher Scientific) according to the manufacturer's instructions. Samples were sequenced on Illumina HiSeq 4000 with $\sim 50$ million/sample SR 50 reads by IGM Genomics Center, University of California, San Diego.

\section{Construction and Refinement of the Flux Balance Model for M. buryatense 5G(B1) in COBRA Toolbox}

To convert the flux balance model for M. buryatense 5G(B1) into the COBRA Toolbox format (Schellenberger et al., 2011) we used the published genome-scale metabolic network of this strain (De la Torre et al., 2015) developed in Pathway Tools (Karp et al., 2010). Subsequently, all zero-flux reactions were removed from the model. The information about zeroflux reactions was extracted from the solution file of the Pathway Tools. We manually extended the COBRA model by adding exchange rates for both nutrients and secreted metabolites as well as by the biomass equation. Moreover, we used the Paint4Net tool (Kostromins and Stalidzans, 2012) 
installed with the COBRA Toolbox to define dead-ends in the model network and to add corresponding exchange rates for them. To standardize reaction and metabolite IDs, we used abbreviations according to BiGG Models ID Specification and Guidelines (King et al., 2016) and KEGG abbreviations for enzymatic reactions for which we could not find corresponding BIGG reactions. To link genes with certain enzymatic reactions we turned to account NCBI Reference Sequence annotation: NZ_KB455575. Moreover, we added missing formulas for metabolites and checked out the model in COBRA Toolbox to ensure all reactions were mass balanced. The model was also extended by the addition of enzymatic reactions (EC 3.1.2.2; 1.3.8.-; 4.2.1.17; 1.1.1.35; 2.3.1.16) for FA degradation via the $\beta$-oxidation pathway and a reaction catalyzed by phosphoketolase (EC 4.1.2.22). Flux balance analysis (FBA) was performed by solving the Linear Programming problem for biomass optimization on the basis of GNU Linear Programming Kit (GLPK) (http://www.gnu.org/software/glpk/) solver in MATLAB. The converted model for $M$. buryatense $5 G(B 1)$ in COBRA format is available on the web-site: http://sci.sdsu.edu/kalyuzhlab/.

\section{Computational Analysis of the RNA-Seq Data}

The genome sequence and annotation files of $M$. buryatense $5 G(B 1)$ were obtained from NCBI (RefSeq accession number GCF_000341735.1). Reads were aligned to the genome sequence using the Rockhopper 2 software system (Tjaden, 2015) with default settings. It is worth noting that the tool reports integral values for analyzed transcriptomic characteristics, even if the underlying value is a decimal. If a read aligns partially to a transcript, it will contribute a fractional amount to the raw count. Rockhopper 2 normalizes each RNAseq data set using upper quartile normalization, because the normalization procedure of the mapped read counts is an essential preprocessing step for differential expression detection (Bullard et al., 2010). To quantify transcript abundance levels, Rockhopper 2 employs a modified RPKM value based on more robust normalizer of upper quartile transcript expression. The normalized data were used to compare gene expression in the wild type AP18 strain vs. the overexpressed farE and $\triangle$ farE strains using the built-in algorithm of DESeq (Anders and Huber, 2010). To determine whether a transcript shows differential expression in data from two any conditions we used a threshold $(q<0.05)$ of $q$-values that are corrected $p$-values to control the false discovery rate using the Benjamini-Hochberg procedure (Benjamini and Hochberg, 1995). Appropriateness of the $q$-value threshold $<0.05$ was verified with a set of housekeeping genes and genes encoding key $\mathrm{C} 1$-enzymes, and those showed no differential expression [for example: 3-hexulose-6-phosphate synthase, q(AP18 vs. $\mathrm{AP} 18 \Delta$ farE $)=0.28, \mathrm{q}(\mathrm{AP} 18+$ pAWP78 vs. AP18::farE $)=$ 0.53 ; pyruvate carboxylase, $\mathrm{q}(\mathrm{AP} 18$ vs. $\mathrm{AP} 18 \Delta$ farE $)=0.41$, $\mathrm{q}(\mathrm{AP} 18+\mathrm{pAWP} 78$ vs. AP18::farE $)=0.64$; arginyl-tRNA-protein transferase, $\mathrm{q}(\mathrm{AP} 18$ vs. AP18 $\Delta$ farE $)=1, \mathrm{q}(\mathrm{AP} 18+\mathrm{pAWP} 78$ vs. AP18::farE) $=1$ ].

\section{RESULTS}

\section{Lipid Biosynthesis and Degradation: M. buryatense 5G(B1) Genome Mining}

Methane metabolism in the $M$. buryatense $5 G(B 1)$ strain is outlined in Figure 1A. The automatic annotation of the genome predicts a typical Type II FA biosynthesis pathway. The FA-biosynthesis genes form several clusters: (1) a plsXfabHDGacpfabF-cluster, which includes genes for a fatty acid/phospholipid synthesis protein, 3-oxoacyl-ACP synthase III, malonyl-CoA-ACP transacylase, 3-oxoacyl-ACP reductase, acyl carrier protein (ACP), and 3-oxoacyl-ACP synthase II. The cluster also includes a hypothetical protein and a $50 \mathrm{~S}$ ribosomal protein L32 gene upstream of plsX. The organization of this cluster is strongly conserved among all methanotrophic bacteria; (2) a fabAB-cluster encoding 3-oxoacyl-ACP synthase I and beta-hydroxydecanoyl thioester dehydrase; (3) a fabGFZ/A cluster, encoding 3-oxoacyl-ACP synthase II, 3-oxoacyl-ACP reductase, and beta-hydroxyacyl-ACP dehydratase; and (4) an acc $C B$-gene cluster encoding biotin carboxylase and biotin carboxyl carrier protein (BCCP). Genes encoding the acetylcoenzyme A carboxylase carboxyltransferase alpha subunit $(\operatorname{acc} A)$ and beta subunit $(a c c D)$, a second copy of the acetyl-CoA carboxylase biotin carboxylase subunit $(a c c C)$, an additional (3R)-hydroxymyristol ACP dehydratase ( $f a b Z$ ), and two genes for ACP are located at sites distant from each other and other FA-biosynthesis genes.

Synthesis of phospholipids in $M$. buryatense $5 G(B 1)$ appears to follow the E. coli paradigm where a phosphatidic acid precursor is synthesized from glycerol 3-phosphate and FA precursors (Yao and Rock, 2012). However, no plsB (Glycerol3-phosphate acyltransferase) homologs were identified within the $M$. buryatense $5 G(B 1)$ genome. Instead, homologs of $p l s X$ and $p l s Y$ were found. $P l s X Y$ has been shown to preferentially utilize acyl-acyl carrier protein and not acyl-CoA as an acyl donor (Lu et al., 2006). A set of phospholipid-modifying enzymes whose homologs have been shown to act on phospholipids post-synthetically were found in the genome. The first of these enzymes is a membrane-bound desaturase, DesC, which shows about $60 \%$ identity to other well-characterized desaturases that catalyze the oxygen-dependent introduction of a double bond at the $\Delta 9$ position of saturated FAs, particularly stearic acid (Zhu et al., 2006; Parsons and Rock, 2013). Additionally, $M$. buryatense $5 G(B 1)$ contains two copies of $c f a$, a cyclopropane FA synthase, which uses S-adenosyl methionine to generate a cyclopropyl group at a double bond position of a FA (Grogan and Cronan, 1997). Cyclopropane FAs have been previously observed in other methanotrophic bacteria (Methylococcus capsulatus, Methylobacterium organophilum) during growth on methane and during oxygen limitation (Patt and Hanson, 1978; Jahnke and Nicholst, 1986). An intriguing aspect of these lipid-modifying enzymes in $M$. buryatense $5 G(B 1)$ is that both copies of $c f a$ and $\operatorname{des} C$ are clustered together as part of a larger operon. Additionally the operon appears to encode a histidine sensor kinase, a lipase/esterase, and a sterol reductase gene. The physiological significance of this is unclear; however, it is tempting to speculate that these various activities are genetically 

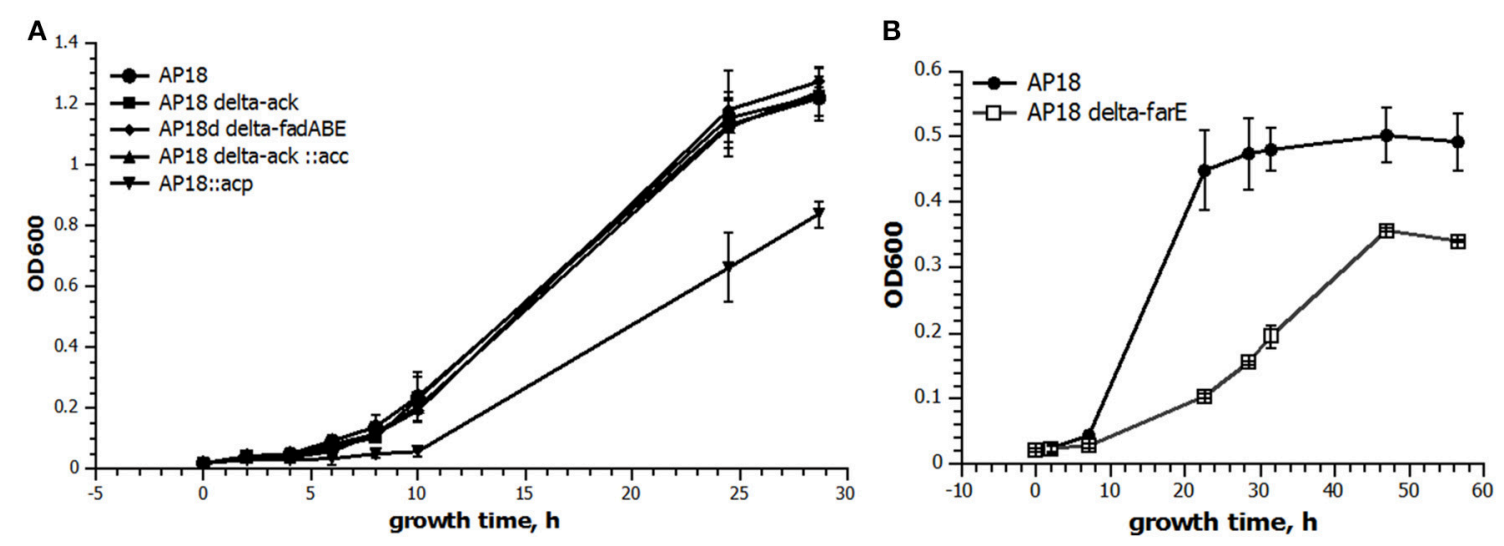

FIGURE 2 | Growth curves of select strains. Twenty milliliters of cultures were grown in serum bottles with $50 \mathrm{ml}$ methane at $30^{\circ} \mathrm{C}$ with shaking for the indicated time. The data are presented as the mean $\pm S D(n=3)$. (A) Comparison of growth rates of the original AP18 (circles) and AP18 $\Delta$ ack (squares), AP18 $\Delta$ fadABE (diamonds), AP18 $\Delta$ ack::acc (triangles), and AP18::acp (inverted triangles). (B) AP18 (filled circles) vs. AP18 $\Delta$ farE (open squares).

linked as an operon for a coordinated membrane remodeling response in $M$. buryatense $5 G(B 1)$. The $M$. buryatense $5 G(B 1)$ genome lacks free fatty transport gene, $f a d L$, but contains an acylCoA synthetase gene, acs $A$, which implies that $M$. buryatense $5 G(B 1)$ cannot transport fatty acids. However, the rest of genes, $f a d A, f a d B$, and $f a d E$, responsible for the degradation and recycling of FAs via $\beta$-oxidation, were identified. The presence of the PlsXY pathway for phospholipids biosynthesis and the lack of a FadD homolog suggest that M. buryatense 5G(B1) is not capable of incorporating exogenous fatty acids into its phospholipid membranes.

Neither fabR/desT nor fadR (a known transcriptional regulators of fatty acid biosynthesis/degradation in E.coli) homologs were detected. The lack of any recognizable transcriptional regulatory factors is unique and could imply that FA biosynthesis in $M$. buryatense $5 G(B 1)$ is being regulated by an as-yet-to-be discovered mechanism. It seems unlikely that FA biosynthesis in methanotrophs is not regulated as formation of ICMs is commensurate with an increase of FA content per cell (Gilman et al., 2015).

\section{Determining Control Points in Fatty Biosynthesis}

The overreaching goal of the study was to identify targets to manipulate fatty acid biosynthesis in methanotrophs. The FBA suggests that about $7.5 \%$ of methane carbon enters the fatty acid biosynthesis pathway. According to genomic data, M. buryatense $5 G(B 1)$ possesses three different metabolic routes for production of acetyl-CoA from single carbon compounds with the main reaction contributing to acetyl-CoA formation predicted to be pyruvate dehydrogenase (Figure 1A). The FBA predictions are consistent with published enzymatic evidence and transcriptomic data (Figure 1; Kalyuzhnaya et al., 1999; De la Torre et al., 2015), as well as with transcriptomic data generated in this study.

ACP is one of the key proteins in FA biosynthesis. In the $M$. buryatense $5 G(B 1)$ genome there are three genes encoding ACP of which only one is highly expressed (Table 4). The differential regulation of $\mathrm{ACP}$ expression raised a question about whether $M$. buryatense $5 G(B 1)$ FA metabolism is saturated with ACP or whether overproduction of ACP would stimulate FA synthesis. If ACP is a limiting factor, its overexpression would lead to an overall increase of the FA production. Alternatively, adverse effects on cell physiology might be observed if cells do not tolerate higher ACP levels. To distinguish between these hypotheses, we chose to overexpress the highly expressed acp gene (WP_014148504.1). An ACP-overexpressing plasmid was constructed and introduced into the AP18 strain. Unlike most other strains tested in this study, the ACP-upregulated strain showed growth defects (Figure 2) and no signs of increased FA production (Table 2, Figure 3). Addition of FabB to the overexpressing ACP strain did not enhance its FAME content. Thus, higher levels of ACP production seem to be toxic for the methanotrophic culture, as seen for E. coli (Keating et al., 1995).

To determine whether FA biosynthesis in methanotrophs is limited by the elongation step, ketoacyl-ACP synthases $(f a b B)$ overexpression was examined. No changes in either the amount of FAME or composition of FAME were observed (Table 2, Figure 3). In order to define the role of $\beta$-oxidation in methanotrophic FA metabolism, the entire cluster of $f a d A$, $f a d B$, and $f a d E$ genes was deleted. The $\triangle f a d(A B E)$ mutant did not show significant changes in growth characteristics (Figure 2); furthermore, the $\triangle \mathrm{fad}(A B E)$-mutant cells had higher levels of FAs (10\% increase compared to original AP18 strain; Table 2, Figure 3).

\section{Improving Carbon Flux into Fatty Acid Building Blocks: Acetyl- and Malonyl-CoA}

Having established that neither ACP nor elongation of FA synthesis are limiting steps in FA accumulation, we then tested the possibility that directing carbon flux into acylCoA and malonyl-CoA pools would have a positive impact on total FA accumulation. $M$. buryatense $5 G(B 1)$ cells excrete acetate $\left(\sim 100 \mu \mathrm{mol} \mathrm{gCDW}^{-1}\right)$ and formate $(\sim 500 \mu \mathrm{mol}$ $\mathrm{gCDW}^{-1}$ ) during growth on methane (Gilman et al., 2015). The 
TABLE 2 | FA methyl ester (FAME) content of the strains used in this study (percent $=$ FAME/dry cell weight).

\begin{tabular}{lcc}
\hline Strain & \multicolumn{1}{c}{ FAME \% } & Increase, folds over AP18 \\
\hline AP18 & $9.17 \pm 0.80$ & 1.00 \\
AP18 $\Delta$ ack & $9.88 \pm 0.72$ & 1.08 \\
AP18 $\Delta$ fadABE & $10.12 \pm 0.89$ & 1.10 \\
AP18::acc & $10.77 \pm 0.18$ & 1.17 \\
AP18 $\Delta$ ack::acc & $11.07 \pm 0.22$ & 1.21 \\
AP18 $\Delta$ fadABE $\Delta$ ack::acc & $10.63 \pm 0.45$ & 1.16 \\
AP18 $\Delta$ farE & $6.71 \pm 0.51$ & 0.73 \\
AP18::fare & $9.14 \pm 0.15$ & 1.00 \\
AP18::fabB & $9.04 \pm 0.19$ & 0.99 \\
AP18::acp & $8.76 \pm 0.43$ & 0.96 \\
AP18::fabB::acp & $8.54 \pm 0.25$ & 0.93 \\
\hline
\end{tabular}

concentrations of excreted organic acids are not high; however, the leak of $\mathrm{C}_{2}$-compounds might indicate an additional loss of carbon needed for FA biosynthesis. In order to increase carbon flow into FA biosynthesis two additional modifications were made: deletion of acetate kinase ( $\triangle a c k$ ) and overexpression of acetyl-CoA carboxylase $(a c c)$. It should be mentioned that the overexpression of acetyl-CoA carboxylase $(\operatorname{acc} A B C D)$ in the $M$. buryatense $5 \mathrm{G}(\mathrm{B} 1)$ strain did not show a statistically significant increase (Puri and Lidstrom, unpublished data). Here, we tested acc-overexpression in different genetic backgrounds. The AP18 $\triangle a c k$ and overproducing Acc strains were found to produce $99 \pm 7$ and $108 \pm 2 \mathrm{mg} / \mathrm{gDCW}$ of FAME, respectively (Table 2, Figure 3). Moreover, when combined together in the same strain, the effects of both mutations were additive resulting in the highest FA levels $(111 \pm 2 \mathrm{mg} / \mathrm{gDCW}, \sim 20 \%$ more than in the original AP18) achieved. Deletion of $f a d A B E$ genes in AP18 $\Delta$ ack::acc background did not increase FAME levels.

\section{Regulation of Fatty Acid Biosynthesis in M. buryatense 5G(B1)}

The $M$. buryatense $5 G(B 1)$ genome does not contain proteins homologous to typical bacterial regulators of FA metabolism, such as $f a b R, f a d R$, or desT. However, an orf (WP_017839568) with a weak homology to the nucleotidebinding Maf family proteins was identified upstream of the plsXfabHDGacpfabF-cluster. Homologous genes were identified in eight methanotrophic genomes ( $M$. buryatense, Methylomicrobium alcaliphilum, Methylomicrobium marinus, Methylomicrobium luteus, $M$. sp $M K$ WGS, M. sp.11b, Methylomicrobium methanica, and $M$. capsulatus), and in each case the gene located upstream of the FA biosynthesis genes. The conserved proximity of the fab-gene suggested it could be linked to FA metabolism. A WP_017839568 deletion mutant was generated and shown to have a severe growth defect (Figure 2). Cellular lipid content was also impaired: not only was the FAME content decreased but also there was an unusually high amount of C18:1 complemented by a decrease in C16:1 (Table 3). Reciprocal up-regulation (overexpression) of the WP_017839568

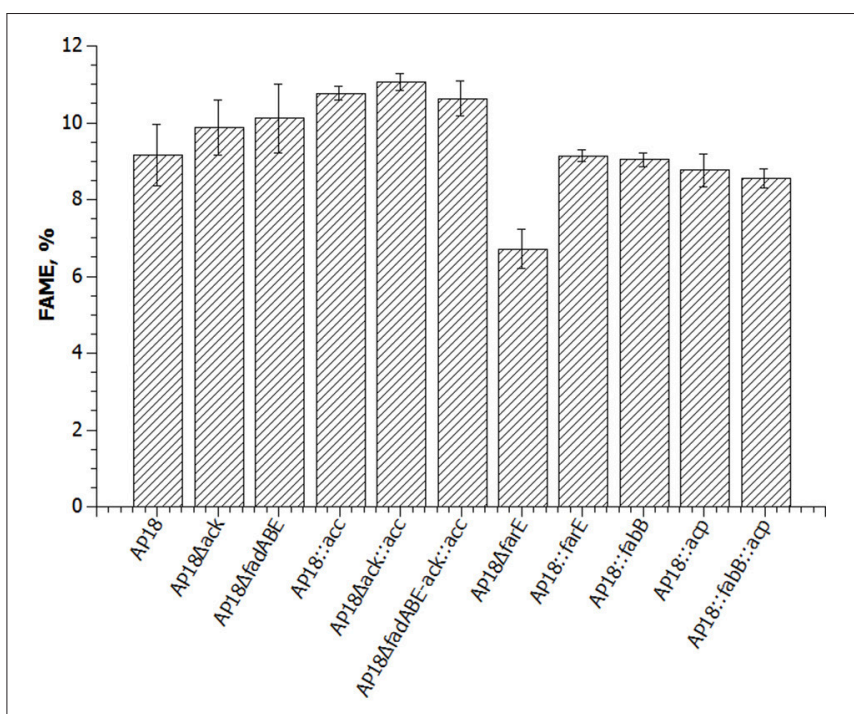

FIGURE 3 | Graphic presentation of FAME levels in the same strains as in Table 2 (percent = FAME/dry cell weight).

gene showed no growth defects and approximately the same FA profile as the initial strain (Table 3).

This striking phenotype of the WP_017839568-deleted strain suggested the possibility that this gene might be involved in the regulation FA synthesis, especially elongation of growing FA chains past 16 carbon atoms. In this regard, we proposed the name farE (for Fatty Acid Regulator of Elongation) for the WP_017839568 gene. In order to investigate the mechanism of such regulation, total RNA sequencing was performed (Table S3) for the following strains: (i) original AP18 strain (ii) AP18 $\triangle$ farE, (iii) AP18::farE, and (iv) AP18::pAWP78 (empty plasmid). By comparison of AP18 to AP18 $\Delta$ farE, 1600 differentially expressed transcripts were identified. The majority of the differentially expressed genes are related to general metabolism and cellular functions (not shown), which could be explained by significant differences in growth rates between wild type and the farE-mutant. A subset of differentially regulated genes upon farE deletion is involved in FA biosynthesis or maintaining the acyl-CoA pool (Table 4). They include all three copies of the acp $P$ gene present in $M$. buryatense $5 G(B 1)$ genome, two copies of the $f a b F$ and $f a b A$ genes, and the $f a b G$ and $f a b D$ genes. Two more insufficiently annotated genes included another copy of 3-ketoacyl-ACP synthase (WP_017841167.1) and a new thioesterase (WP_040575583.1, acyl-CoA thioester hydrolase). Considering their function in elongation of FA biosynthesis, upregulation of either 3-ketoacyl-ACP synthase (two copies of fabF and WP_017841167.1) could be a logical explanation for the elevated levels of the normally uncommon C18 FA in $M$. buryatense $5 G(B 1)$. Upon deletion of farE, the Acp expression pattern changes so that all three acp $P$ genes are slightly upregulated (1.32x, 1.75x, and 2.13x).

In order to further narrow down the list of potential candidates for explaining changes in the $\triangle$ farE mutant, we compiled a table (Table 5) including only those FA 
TABLE 3 | FA profile of original AP18 and strains with up- and down-regulated farE.

\begin{tabular}{|c|c|c|c|c|c|c|c|}
\hline \multirow[t]{2}{*}{ Strain } & \multicolumn{7}{|c|}{ Fatty acid, mg/gDcW } \\
\hline & C14:0 & C15:0 & C16:1n7 & C16:1n9 & C16:0 & C18:1 & C18:0 \\
\hline AP18, replicate 1 & 3.23 & 0.48 & 55.38 & 12.07 & 14.95 & 0.25 & 0.30 \\
\hline AP18, replicate 2 & 3.32 & 0.40 & 50.09 & 9.17 & 13.39 & 0.27 & 0.20 \\
\hline AP18 $\Delta$ faeR, replicate 1 & 0.00 & 0.00 & 23.49 & 6.24 & 8.70 & 24.06 & 0.97 \\
\hline AP18 $\Delta$ faeR, replicate 2 & 0.00 & 0.23 & 23.84 & 7.73 & 9.05 & 28.09 & 1.72 \\
\hline AP18::faeR, replicate 1 & 4.53 & 0.62 & 54.34 & 15.36 & 16.89 & 0.24 & 0.49 \\
\hline AP18::faeR, replicate 2 & 4.25 & 0.59 & 52.55 & 14.93 & 17.31 & 0.22 & 0.54 \\
\hline
\end{tabular}

TABLE 4 | Differentially regulated genes of FA biosynthesis with down- and up-regulated farE compared to original AP18 ( $q<0.05$ ).

\begin{tabular}{|c|c|c|c|c|c|}
\hline & \multirow[t]{2}{*}{ Gene ID } & \multirow[t]{2}{*}{ Gene function } & \multicolumn{3}{|c|}{ Expression } \\
\hline & & & AP18 & AP18 $\Delta$ farE & AP18::farE \\
\hline fabA & WP_014149549.1 & 3-Hydroxyacyl-ACP dehydratase & 89 & 102 & 200 \\
\hline \multirow[t]{2}{*}{ fabA } & WP_017839698.1 & 3-Hydroxydecanoyl-ACP dehydratase; & 121 & 109 & 144 \\
\hline & WP_017841166.1 & 3-Hydroxyacyl-ACP dehydratase & 39 & 60 & 31 \\
\hline fabG & WP_017841165.1 & 3-Ketoacyl-ACP reductase & 33 & 109 & 38 \\
\hline- & WP_017841167.1 & 3-Ketoacyl-ACP synthase & 29 & 61 & 32 \\
\hline fabF & WP_026130034.1 & 3-Oxoacyl-ACP synthase & 166 & 162 & 291 \\
\hline fabF & WP_026130222.1 & 3-Oxoacyl-ACP synthase & 40 & 54 & 38 \\
\hline- & WP_017840388.1 & Acetyl-CoA carboxylase subunit alpha & 73 & 82 & 56 \\
\hline acpP & WP_014148504.1 & Acyl carrier protein & 1136 & 1505 & 1733 \\
\hline acpP & WP_017841105.1 & Acyl carrier protein & 29 & 51 & 41 \\
\hline acpP & WP_017841106.1 & Acyl carrier protein & 62 & 132 & 65 \\
\hline- & WP_040575583.1 & acyl-CoA thioester hydrolase & 25 & 42 & 33 \\
\hline \multirow[t]{2}{*}{ fabD } & WP_017839572.1 & Malonyl CoA-ACP transacylase & 391 & 847 & 469 \\
\hline & WP_017840721.1 & 2-Isopropylmalate synthase & 113 & 108 & 97 \\
\hline farE & WP_017839568 & Fatty acid regulator of elongation & 29 & 4 & 1658 \\
\hline
\end{tabular}

Calculated normalized transcript abundance levels are show.

biosynthesis-related genes that are regulated in opposite directions in $\triangle$ farE and farE overexpression strains. In addition, genes differentially regulated in AP18 and AP18::pAWP78 (empty plasmid) were also removed. That allowed us to narrow the list to only three entries (FabF, WP_026130034.1; 3-hydroxydecanoyl-ACP dehydratase, WP_017839698.1; 2isopropylmalate synthase, WP_017840721.1). Effects of up- and down-regulation of these genes will be tested in future studies.

\section{DISCUSSION}

Microbial fatty acid biosynthesis pathways are among the most attractive targets for the production of fuels and oleochemicals from renewable feedstocks (Lennen and Pfleger, 2013; Janßen and Steinbuchel, 2014). Different microbial systems as well as numerous metabolic engineering strategies have been explored for improving biofuel production (Lennen and Pfleger, 2013). The most common approaches aim to reduce the catabolism of fatty acids (e.g., deletion of $f a d B A E$ or $f a d R$ genes), to eliminate feedback inhibition in the biosynthetic pathway (e.g., overexpression of $\operatorname{acc} A B C D$, fabD with/without thioesterase I (tes $A)$ genes and/or deletion of $f a b R$ ), as well as incorporating heterologous enzymes or metabolic pathways for converting the FA intermediates into free fatty acids, fatty acid methyl esters, and fatty acid ethyl esters, olifins, etc. (Clomburg and Gonzales, 2010; Lennen and Pfleger, 2013; Janßen and Steinbuchel, 2014).

Core metabolic functions of many methanotrophic bacteria are interlinked with the development of intracytoplasmic membranes. As a result, methanotrophic bacteria have relatively high lipid/biomass content (9-10\% of dry weight, mostly as phospholipids). Here, we investigated fatty acid biosynthesis in $M$. buryatense $5 G(B 1)$. Overall, the metabolic pathways for fatty acid production and degradation in $M$. buryatense $5 G(B 1)$ resemble the canonical bacterial pathways with only a few exceptions, such as the lack of recognizable fadD/fadL and regulators. Absence of the $f a d L$ gene is not surprising since $M$. buryatense $5 G(B 1)$ is known for not being able to utilize long-chain FAs as a carbon or energy source. On the other hand, the absence of $f a d D$, the mechanism by which free FAs becomes activated (i.e., ligation to CoA) for beta oxidation is puzzling. The phenotype of fadABE mutants indicates that betaoxidation pathway is active in $M$. buryatense $5 G(B 1)$. A set of 
TABLE 5 | Opposite directions* of differentially expressed genes in two datasets: original AP18 vs. down-regulated farE and AP18+pAWP78 compared to up-regulated farE.

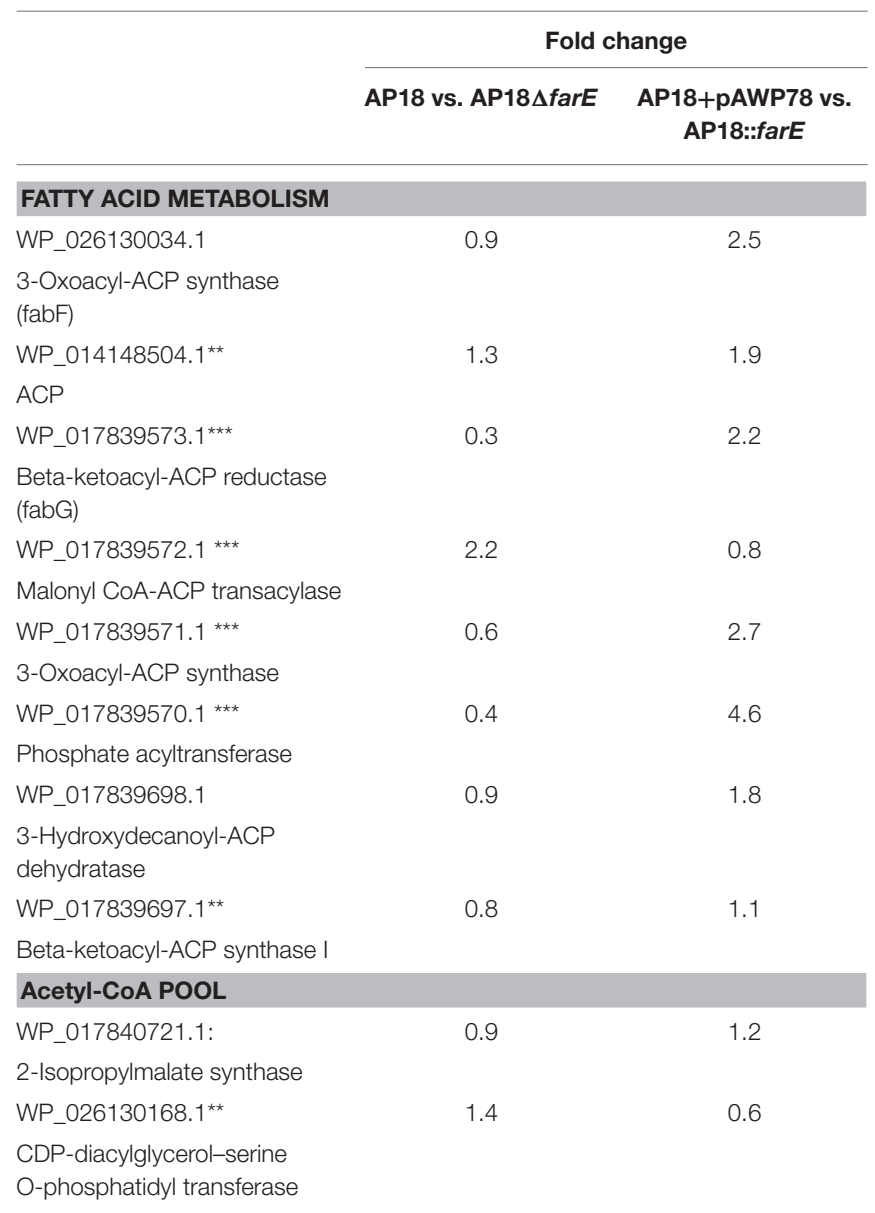

${ }^{*}$ Down-regulated in AP18 vs. AP18 farE and up-regulated in AP18+pAWP78 vs. AP18::fare;

**Neighboring gene; ***Neighboring genes from one gene cluster.

genes annotated as Acyl-CoA synthetases were identified in the genome, each of them might potentially fulfill the fadD function.

Given that intra-cytoplasmic membranes are primarily produced during growth on methane and that a membranebound methane monooxygenase, pMMO, is associated with these membranes, a regulatory mechanism must exist to repress FA biosynthesis during growth on non-methane carbon sources (i.e., methanol). The initial hypothesis posed that farE could encode a FA metabolism regulator. Our results support this hypothesis. farE deletion changes expression of many metabolic genes including several involved in FA biosynthesis; the FA content of the mutant is also drastically changed. The phenotypic feature of AP18 $\triangle$ farE, i.e., conversion of a significant portion of C16 FA into C18 FA, could be potentially explained by overproduction of 3 FA synthesis elongation enzymes, two fabF genes and one less characterized 3-ketoacyl-ACP synthase gene (WP_017841167.1). Another option for accumulation of C18 FAs could be overregulated acpP genes. Only one of the 3 acpP genes is highly transcribed (WP_014148504.1); the two others are transcribed in low amounts. However, these low-expressed copies of acyl carrier proteins could have different properties from the main one resulting in synthesis of longer FAs. It has been recently demonstrated that the size of the Acp hydrophobic pocket influences the length of FA synthesized, with smaller pockets leading to cellular accumulation of shorter FAs (Liu et al., 2016). In our case we would expect that one of the Acp copies would have a bigger hydrophobic pocket resulting in synthesis of longer lipids. In addition, FarE contributed to upregulation of a number FA biosynthesis genes, including fabA (hydroxyacyl-ACP dehydratase), fabG (3-ketoacyl-ACP reductase), and thioesterase. Overall, it could be suggested that FarE acts as a regulator of fatty acid metabolism, although there is a possibility that the observed AP18 $\Delta$ farE phenotype could be achieved through indirect action of unidentified regulatory mechanisms.

A set of different strategies was tested for improving fatty acid production in $M$. buryatense $5 G(B 1)$. While the overexpression of the $f a b B$ did not alter the FA composition significantly, the modulation of FA synthesis elongation seems to be a viable strategy to manipulate the FA content in methanotrophs. Analysis of transcriptomics data of the AP18 $\Delta$ farE strain suggested three potential candidates for synthesis of longer-thanusual C18 FA in $M$. buryatense $5 G(B 1)$. These proteins with $3-$ ketoacyl-ACP synthase (KAS) activities comprise $2 \mathrm{fabF}$ genes and one previously unidentified KAS (WP_017841167.1) gene. Previously, $f a b F$ was identified as an activator of FA biosynthesis in E. coli (Lee et al., 2013), which makes it attractive target for biotechnological manipulation. Another difference is the ability of $f a b F$ but not $f a b B$ to elongate palmitoleic acid (C16:1) for synthesis of a C18:1 version (Edwards et al., 1997). However, whether the increased activity of $f a b F$ would be sufficient for switching of FA biosynthesis from predominantly C16 FA to C18 remains a question. In this regard, the third KAS enzyme (WP_017841167.1), which is still uncharacterized, could also be considered. Whether overexpression of any of them would result in changes in FA profile will be accessed in future studies.

It has been previously observed that overexpression of acp $P$ from a multi-copy plasmid leads to inhibition of cell growth in E. coli (Keating et al., 1995). That effect was attributed to an unmodified (Apo) form of acyl carrier protein since overexpressed ACP was incompletely post-translationally modified and accumulation of apo-Acp resulted in an inability to transfer the completed FA to glycerol 3-phosphate. However, lower levels of Acp, including AcpS from other organisms, could be produced in E. coli without toxicity (Guerra et al., 1988). Since the overexpression plasmid used in the current study (pAWP78, Puri et al., 2015) is a medium- to low-copy number plasmid, we did not expect inhibitory effects of Acp overproduction in $M$. buryatense $5 G(B 1)$. However, adverse effects resulting in inhibition of growth upon overproduction of Acp were observed indicating that the mechanism of post-translation modification of Acp by holo-ACP synthase is adjusted to regulate Acp levels and might be inefficient when Acp is overproduced. Further attempts to upregulate FA accumulation by Acp overproduction should be supported by simultaneous overproduction of holoACP synthase. 
The genetic alterations of fatty acid turnover as well as key elements of fatty acid biosynthesis resulted in very minor changes in overall lipid content, suggesting the capacity of the system is restricted by the overall influx of carbon into the pathway. However, strains with ack deleted (block in acetyl-CoA to acetate conversion) showed an increased FA content. Moreover, a strain with both $\triangle a c k$ and overexpression of acc had the highest FAME content among all tested in this study. The inconsistences in acc-overexpression outcomes could be explained by insufficient biotinylation of the protein.

\section{SUMMARY}

Metabolic pathways for fatty acid biosynthesis are reconstructed and a set of novel mechanisms contributing to FA-biosynthesis regulation and metabolic precursor formation are proposed. $M$. buryatense $5 G(B 1)$ naturally accumulates more FA than many other bacteria in order to maintain high amounts ICM structures (8.8\% FAME content). In this work, several strategies were demonstrated to increase FA production in methanotrophic bacteria further (resulting up to 11\% FAME content per dry cell weight). The tested strategies, despite targeting different nonoverlapping pathways, were not usually additive suggesting the

\section{REFERENCES}

Anders, S., and Huber, W. (2010). Differential expression analysis for sequence count data. Genome Biol. 11, 1-28. doi: 10.1186/gb-2010-1110-r106

Benjamini, Y., and Hochberg, Y. (1995). Controlling the false discovery rate: a practical and powerful approach to multiple testing. J. R. Statist. Soc. Ser. B 57, 289-300.

Bullard, J. H., Purdom, E., Hansen, K. D., and Dudoit, S. (2010). Evaluation of statistical methods for normalization and differential expression in mRNA-Seq experiments. BMC Bioinform. 11:94. doi: 10.1186/1471-2105-11-94

Chu, F., Beck, D., and Lidstrom, M. E. (2016). MxaY regulates the lanthanidemediated methanol dehydrogenase switch in Methylomicrobium buryatense. PeerJ 4:e2435. doi: 10.7717/peerj.2435

Chu, F., and Lidstrom, M. E. (2016). XoxF Acts as the predominant methanol dehydrogenase in the type I methanotroph Methylomicrobium buryatense. J. Bacteriol. 198, 1317-1325. doi: 10.1128/JB.00959-15

Clomburg, J. M., and Gonzales, R. (2010). Biofuel production in Escherichia coli: the role of metabolic engineering and synthetic biology. Appl. Microbiol. Biotechnol. 86, 419-434. doi: 10.1007/s00253-010-2446-1

De la Torre, A., Metivier, A., Chu, F., Laurens, L., Beck, D., Pienkos, P., et al. (2015). Genome-Scale metabolic reconstructions and theoretical investigation of methane conversion in Methylomicrobium buryatense strain 5G(B1). Microb. Cell Fact. 14, 188. doi: 10.1186/s12934-015-0377-3

Edwards, P., Nelsen, J. S., Metz, J. G., and Dehesh, K. (1997). Cloning of the fabF gene in an expression vector and in vitro characterization of recombinant fabF and fabB encoded enzymes from Escherichia coli. FEBS Lett. 402, 62-66. doi: 10.1016/S0014-5793(96)01437-8

Gilman, A., Laurens, L., Puri, A., Chu, F., Pienkos, P., and Lidstrom, M. E. (2015). Bioreactor performance parameters for an industrially-promising methanotroph Methylomicrobium buryatense 5GB1. Microb. Cell Fact. 14, 182. doi: 10.1186/s12934-015-0372-8

Griffiths, R. I., Whiteley, A. S., O'Donnell, A. G., and Bailey, M. J. (2000). Rapid method for coextraction of DNA and RNA from natural environments for analysis of ribosomal DNA- and rRNA-based microbial community composition. Appl. Environ. Microbiol. 66, 5488-5491. doi: 10.1128/AEM.66.12.5488-5491.2000 existence of a yet-to-be discovered mechanism of regulation of FA levels.

\section{AUTHOR CONTRIBUTIONS}

$\mathrm{AD}, \mathrm{EA}$, and $\mathrm{MK}$ designed the experiments and coordinated the study. $\mathrm{AD}$ carried out all genetic, growth, and transcriptomic experiments and analyzed data. $\mathrm{AD}$ and MA performed genome mining and wrote the first draft of the manuscript. IA contributed to model construction and RNA-data analyses. MK and EA helped to analyze the data and write the manuscript. All authors reviewed and approved the final manuscript.

\section{ACKNOWLEDGMENTS}

This work was funded by grants from ARPA-E (DE-AR0000350 and DE-AR0000438).

\section{SUPPLEMENTARY MATERIAL}

The Supplementary Material for this article can be found online at: http://journal.frontiersin.org/article/10.3389/fmicb. 2016.02167/full\#supplementary-material

Grogan, D. W., and Cronan, J. E. (1997). Cyclopropane ring formation in membrane lipids of bacteria. Microbiol. Mol. Biol. Rev. 61, 429-441.

Guerra, D. J., Dziewanowska, K., Ohlrogge, J. B., and Beremand, P. D. (1988). Purification and characterization of recombinant spinach acyl carrier protein I expressed in Escherichia coli. J. Biol. Chem. 263, 4386-4391.

Henard, C., Smith, H., Dowe, N., Kalyuzhnaya, M., Pienkos, P., and Guarnieri, M. (2016). Bioconversion of methane to lactate by an obligate methanotrophic bacterium. Sci. Rep. 6:21585. doi: 10.1038/srep21585

Jahnke, L., and Nicholst, P. (1986). Methyl sterol and cyclopropane fatty acid composition of Methylococcus capsulatus grown at low oxygen tensions. methyl sterol and cyclopropane fatty acid composition of Methylococcus capsulatus grown at low oxygen tensions. J. Bacteriol. 167, 238-242. doi: 10.1128/jb.167.1.238-242.1986

Janßen, H. J., and Steinbuchel, A. (2014). Fatty acid synthesis in Escherichia coli and its applications towards the production of fatty acid based biofuels. Biotechnol. Biofuel. 7:7. doi: 10.1186/1754-6834-7-7

Kaluzhnaya, M., Khmelenina, V., Eshinimaev, B., Suzina, N., Nikitin, D., Solonin, A., et al. (2001). Taxonomic characterization of new alkaliphilic and alkalitolerant methanotrophs from soda lakes of the southeastern transbaikal region and description of Methylomicrobium buryatense sp. nov. Syst. Appl. Microbiol. 24, 166-176. doi: 10.1078/0723-2020-00028

Kalyuzhnaya, M. G., Khmelenina, V. N., Lysenko, A. M., Suzina, N. E., Trotsenko, Y. A. (1999). New methanotrophic isolates from soda lakes of the southern Transbaikal region. Mikrobiologiia 68, 689-697.

Karp, P. D., Paley, S. M., Krummenacker, M., Latendresse, M., Dale, J. M., Lee, T. J., et al. (2010). Pathway tools version 13.0: integrated software for pathway/genome informatics and systems biology. Brief. Bioinform. 11, 40-79. doi: 10.1093/bib/bbp043

Keating, D. H., Carey, M. R., and Cronan, J. E. (1995). The unmodified (Apo) form of Escherichia coli acyl carrier protein is a potent inhibitor of cell growth. J. Biol. Chem. 270, 22229-22235. doi: 10.1074/jbc.270.38.22229

Khmelenina, V. N., and Beck, D. A. C. (2013). Draft genome sequence of Methylomicrobium buryatense strain 5G, a haloalkalinetolerant Methanotrophic bacterium. Genome Announc. 1, 16-17. doi: 10.1128/genomeA.00053-13

King, Z. A., Lu, J., Dräger, A., Miller, P., Federowicz, S., Lerman, J. et al. (2016). BiGG models: a platform for integrating, standardizing and sharing 
genome-scale models. Nucleic Acids Res. 44, D515-D522. doi: 10.1093/nar/ gkv1049

Kostromins, A., and Stalidzans, E. (2012). Paint4Net: COBRA toolbox extension for visualization of stoichiometric models of metabolism. BioSystems 109, 233-239. doi: 10.1016/j.biosystems.2012.03.002

Lee, S., Lee, S., Yoon, Y. J., and Lee, J. (2013). Enhancement of long-chain fatty acid production in Escherichia coli by coexpressing genes, including fabF, involved in the elongation cycle of fatty acid biosynthesis. Appl. Biochem. Biotechnol. 169, 462-476. doi: 10.1007/s12010-012-9987-y

Lennen, R. M. and Pfleger, B. F. (2012). Engineering Escherichia coli to synthesize free fatty acids. Trends Biotechnol. 30, 659-667. doi: 10.1016/j.tibtech.2012.09.006

Lennen, R. M., and Pfleger, B. F. (2013). Microbial production of fatty acid-derived fuels and chemicals. Curr. Opin. Biotechnol. 24, 1044-1053. doi: 10.1016/j.copbio.2013.02.028

Levett, I., Birkett, G., Davies, N., Bell, A., Langford, A., Laycock, B. et al. (2016). Techno-economic assessment of Poly-3-Hydroxybutyrate (PHB) production from methane - the case for thermophilic bioprocessing. J. Environ. Chem. Eng. 4, 3724-3733. doi: 10.1016/j.jece.2016.07.033

Liu, X., Hicks, W., Silver, P., and Way, J. C. (2016). Engineering acyl carrier protein to enhance production of shortened fatty acids. Biotechnol. Biofuels 9 , 24. doi: 10.1186/s13068-016-0430-4

Lu, Y., Zhang, Y., Grimes, K., Qi, J., Lee, R., and Rock, C. (2006). Acyl-phosphates initiate membrane phospholipid synthesis in gram-positive pathogens. Mol. Cell 23, 765-772. doi: 10.1016/j.molcel.2006.06.030

Marx, C. J. (2008). Development of a broad-host-range sacB-based vector for unmarked allelic exchange. BMC Res. Notes 1:1. doi: 10.1186/17560500-1-1

Marx, C. J., and Lidstrom, M. E. (2002). Broad-host-range cre-lox system for antibiotic marker recycling in gram-negative bacteria. BioTechniques 33, 1062-1067.

Ojala, D., Beck, D., and Kalyuzhnaya, M. (2011). Genetic systems for moderately halo (Alkali)philic bacteria of the genus Methylomicrobium. Meth. Enzymol. 495, 99-118. doi: 10.1016/B978-0-12-386905-0.00007-3

Parsons, J. B., and Rock, C. O. (2013). Bacterial lipids: metabolism and membrane homeostasis. Prog. Lipid Res. 52, 249-276. doi: 10.1016/j.plipres.2013. 02.002
Patt, T. E., and Hanson, R. S. (1978). Intracytoplasmic membrane, phospholipid, and sterol content of Methylobacterium organophilum cells grown under different conditions. J. Bacteriol. 134, 636-644.

Puri, A., Owen, S., Chu, F., Chavkin, T., Beck, D., Kalyuzhnaya, M., et al. (2015). Genetic tools for the industrially promising methanotroph Methylomicrobium buryatense. Appl. Environ. Microbiol. 81, 1775-1781. doi: 10.1128/AEM.03795-14

Schellenberger, J., Que, R., Fleming, R., Thiele, I., and Orth, J. (2011). Quantitative prediction of cellular metabolism with constraint-based models: the COBRA toolbox v2.0. Nat. Protoc. 6, 1290-1307. doi: 10.1038/nprot.2011.308

Sessions, A. L., Jahnke, L. L., Schimmelmann, A., and Hayes, J. M. (2002). Hydrogen isotope fractionation in lipids of the methane-oxidizing bacterium Methylococcus capsulatus. Geochim. Cosmochim. Acta 66, 3955-3969. doi: 10.1016/s0016-7037(02)00981-x

Strong, P. J., Kalyuzhnaya, M., Silverman, J., and Clarke, W. P. (2016). A methanotroph-based biorefinery: potential scenarios for generating multiple products from a single fermentation. Bioresour. Technol. 215, 314-323. doi: 10.1016/j.biortech.2016.04.099

Tjaden, B. (2015). De Novo assembly of bacterial transcriptomes from RNA-Seq data. Genome Biol. 16:1. doi: 10.1186/s13059-014-0572-2

Yao, J., and Rock, C. (2012). Phosphatidic acid synthesis in bacteria. Biochim. Biophys. Acta 1831, 495-502. doi: 10.1016/j.bbalip.2012.08.018

Zhu, K., Choi, K., Schweizer, H., Rock, C., and Zhang, Y. M. (2006). Two aerobic pathways for the formation of unsaturated fatty acids in Pseudomonas aeruginosa. Mol. Microbiol. 60, 260-273. doi: 10.1111/j.1365-2958.2006.05088.x

Conflict of Interest Statement: The authors declare that the research was conducted in the absence of any commercial or financial relationships that could be construed as a potential conflict of interest.

Copyright (c) 2017 Demidenko, Akberdin, Allemann, Allen and Kalyuzhnaya. This is an open-access article distributed under the terms of the Creative Commons Attribution License (CC BY). The use, distribution or reproduction in other forums is permitted, provided the original author(s) or licensor are credited and that the original publication in this journal is cited, in accordance with accepted academic practice. No use, distribution or reproduction is permitted which does not comply with these terms. 